\title{
The Evolution of Physics: From Aristotle to Stephen Hawking
}

\author{
S.Prasanna $^{1}$, V.S.Venkatavaradhan ${ }^{2}$ \\ \{spa.phy@psgtech.ac.in ${ }^{1}$ drrvsv@yahoo.co.in $\left.{ }^{2}\right\}$ \\ Dept of Physics, PSG College of Technology ${ }^{1}$, Coimbatore, India , formerly, Director, Nehru \\ Science Planetarium, Mumbai, India ${ }^{2}$
}

\begin{abstract}
Science or Physics had its beginnings in some remote era when people began to show curiosity concerning their environment and to record what they saw. In due course of time, reflections on these observations led to the idea that nature is knowable and dependable and that as a result predictions could be made which would give man a certain degree of control over his environment. This article highlights the significant developments of science or specifically Physics since the onset of meaningful civilization. This article will summarize the evolution of Physics from its infancy and look at the contributions of different scientists who have made this journey possible to the present state of existence.
\end{abstract}

Keywords: Quantum, Electrodynamics, Physics, Agriculture.

\section{Origin and rise of science (Physics): Aristotle and Archimedes}

The actual birth of science took place in prehistoric times. Excavations from the remains of great civilizations such as Egypt, Indus valley and Babylon suggest the presence of scientific culture. Earliest and probably the first discoveries in science were 'the wheel', control of fire and organized agriculture. However true progress in science did not begin until about the 6th century BC when Greek civilization was beginning to show up.

It was Aristotle (384 - 322 BC), the Greek philosopher who laid the foundations for the scientific outlook on questions about nature. His influence in many fields, including politics, ethics, rhetoric and metaphysics and of course science was still in evidence 20 centuries after his time. One of Aristotle's greatest contributions to knowledge was his promotion of inductive reasoning. Then came Archimedes and discovered some of the fundamental laws governing the mechanism of floating bodies and applied them to a variety of practical problems. To Archimedes we owe the first applications of mathematics to the description of nature. He was very well-ahead of his time.

\section{The renaissance and aftermath}

This turbulent period was marked by intense intellectual activity. Men like Copernicus, Kepler, Galileo and Newton completely liberated science from the domination of Aristotelian thought and introduced the modern scientific outlook based on observation and experiment. 
The first among the achievements of renaissance was the heleo centric theory advocated by Copernicus. Copernicus reckoned that a much simpler interpretation of planetary motion could be realized by adopting the heleo centric approach.

It is generally admitted that the true spirit of modern scientific Enquirer had its first clear expression in the work of Galileo Galili. Galileo himself developed the basic ideas of velocity, acceleration, inertia and established the laws of falling bodies and projectiles, by a remarkable combination of reasoning and experiments. He was primarily responsible for the fabrication and design of the first astronomical telescope.

A child's first task is to walk and talk and understand his little universe. Newton the 17th century prodigy simply enlarged the project [2]. Newton began assembling and perfecting the Newtonian universe, a miraculously predictable and rational clockwork creation held together by his universal gravitation and regulated by his elegant laws of motion. Newton had a phenomenal list of achievements during his early days. In 1665, he developed his own set of fluxions and in the next year had the theory of colours. His immortal work principia is rated by many as the best ever contribution in the field of science.

During the later half of nineteenth century, physics was more or less considered to be complete and the popular scientists of the day were anxious to add the finishing touches. They imagined that space was filled everywhere by a continuous medium called the ether [1]. Light and other electromagnetic signals were waves in this ether just as sound is pressure waves in air. All that was needed to complete the theory was careful measurements of the elastic properties of the ether. Two scientists Michelson and Morley working on the ether theory meticulously compared the speed of light in two beams at right angles to each other. They reasoned that, as the earth rotates on its axis and orbits the sun, it will move through the ether, and the speed of light in these two beams should diverge. But Michelson and Morley found that light always traveled at the same speed relative to you, no matter how you were moving [1].

It was a young clerk named Albert Einstein; working in the Swiss patent office who cut through the mystery of ether and once for all resolved the speed- of-light problem. In June 1905, he wrote one of three papers that would establish him as on the world's leading scientists and eventually herald into two conceptual revolutions that has forever changed our understanding of space, time and reality [1]. In the 1905 paper, Einstein pointed out that because you could not detect whether or not you were moving through the ether, whole notion of the laws of science should appear the same for all freely moving observers. This broke the concept of universal time.

Any biographical sketch of Albert Einstein will be an understatement. It is difficult for mortal human beings like us to illustrate or narrate the breadth and depth of Einstein's contribution. Richard Feynman, another great scientist had this to say about Einstein "it is impossible for one to believe how he predicted things just by sheer thought" [1-2]. Einstein upset the entire world with his three seminal papers on Brownian motion, photo electricity and relativity. Surely, year 1905 was made for Einstein. The entire human race was not just surprised but stunned by Einstein's prediction. Einstein became a celebrity and was lavishly quoted by the entire human population - scientists, teachers, students, saints and sinners. 
Albert Einstein was a major contributor towards the understanding of photo electricity and special theory of relativity; he single-handedly developed the general theory of relativity, which is essentially a theory of gravity. The experimental evidence for general theory of relativity came in the year 1919 when Astrophysicist Sir Arthur Eddington and his team in South Africa studied the apparent position of stars and made experimental observations, which amazingly coincided with Einstein's theoretical predictions.

Befittingly, Einstein was awarded the Nobel Prize in physics in the year 1921 [5]; it is an irony that the award was not presented to Einstein for his work on relativity, the entire physics world was then not certain about the authenticity of relativity and the royal Swedish academy bestowed this honor upon Einstein for his other contribution - photoelectric effect [1-2].

A new branch of physics - physics of the small, took off during the early half of twentieth century the ideas of Max plank were extended by. Einstein and he laid the foundation for quantum mechanics, ironically he was never comfortable with quantum mechanics and famously asserted, "god does not play dice" but he was proved wrong and eventually evidence poured suggesting that god is very good at playing dice too [2].

Men like Neils Bohr, Heisenberg, Schrödinger and many others immensely contributed to the development of quantum mechanics. Heisenberg developed his own flavour of quantum mechanics by matrix method; Schrödinger, an Austrian by using differential equations, developed an equivalent approach. Of course, Schrödinger's equations were not just famous and good enough to fetch him a Nobel Prize, but were printed in the postage stamps of Austria to acknowledge his contribution [2].

\section{Physics after Einstein}

If General theory of relativity is the greatest theory of 20th century, then the second best theory of course will have to be the theory of quantum electrodynamics (QED). QED deals with inter-atomic interaction. Three scientists Schwinger, Tomonaga and Richard Feynman developed the theory independently, for which they were awarded the Nobel Prize in Physics during 1965. In fact, Richard Feynman, the famous American physicist, popularized QED. Richard Feynman called QED as the "Jewel of Physics"[3]. Richard Feynman was arguably the greatest Physics teachers of modern times, his invaluable lectures on physics, compiled into three volumes, inspired and continues to inspire a generation of physicists. Hans Bethe another Nobel laureate of CornellUniversity once remarked, "There were two kinds of geniuses. The ordinary kind does great things but lets other scientists feel that they could do the same if only they worked hard enough. The other kind performs magic. "A magician does things that nobody else could ever do; and Feynman is a magician" [4].

Physics retains its charm and beauty not just it is fundamental but it is still incomplete. The origin and evolution of our universe is still a fundamental question to be resolved. The big bang theory, which predicts the sequence of events starting from the very creation of our universe, fails to answer the question of the origin itself. As of now, the two corner stones of physics i.e., general theory of relativity and quantum mechanics are miserably at loggerheads with each other. Another great physicist who tried to resolve this impasse was Stephen hawking. Hawking was the St. Lucian professor of mathematics at Cambridge University, a 
position previously held by Isaac Newton and Paul Dirac. Despite his illness, the contribution of Hawking towards physics and science is outstanding. Hawking became an international celebrity since the publication of his immortal book "A Brief history of time". The book remained in the New York Times bestseller list for a period of five years and has sold more than 9 million copies. Stephen hawking resolved the mystery surrounding the black holes and predicted black holes do emit radiation, he developed the theory of quantum black holes and postulated the famous Hawking-Beckenstein radiation [5]

What science or physics has to offer in the next few decades is interesting and bewildering. Can the scientists of the future generation with the advent of artificial intelligence and power of computing be able to solve some of the perplexing fundamental problems? For instance how to bridge quantum mechanics and gravity? It is estimated that a particle accelerator exceeding the size of Milky Way galaxy is required to carry out experiments related to the problem of quantum gravity! Another classical problem waiting to get solved is the problem of turbulence. One has to believe or suspect that accurately predicting the behavior of a phenomenon like weather is almost impossible. The universe is believed to comprise $\sim 5 \%$ normal matter, $\sim 27 \%$ dark matter and $\sim 68 \%$ dark energy [6]. Currently Physicists are certain about what dark matter is not, rather than what it is! The idea of the dark matter is central towards understanding the accelerating expansion rate of our universe. The very word dark implies that it is essentially what we cannot see unlike planets or stars. Dark matter differs from the conventional baryonic matter as they cannot absorb or deflect radiation. [6] Also, it is not anti-matter as anti-matter when annihilated with matter will yield gamma ray bursts which remain to be elusive in this case. One explanation for dark matter is it comprises of some new exotic non-baryonic elementary particle that somehow survived annihilation in the early universe in great numbers to make up the present rest-mass density of the universe. Another dark component of this universe is dark energy having properties very different from those of dark matter. The influence of dark matter on the accelerating universe is governed by the attractive force of gravity. However, the influence of dark energy on the expanding and accelerating universe depends on the dark-energy density and dark-energy pressure. A proper understanding of dark-energy may even lead to the rebirth of Einstein's famous cosmological constant or in other words we may need to get used to a form of repulsive gravity hitherto unseen. Addressing the issues related to dark matter and dark energy presents immense challenges. However solving them would lead a much deeper understanding of our universe and its eventual fate [6-7].

\section{Conclusion}

Physics shall remain largely incomplete until some of these fundamental questions remain unanswered. One fine day we may even be able to appreciate how our universe came into existence but as Stephen Hawking once popularly remarked "We may understand why universe exists? But we shall never understand why it bothers to exist?"

\section{References}

[1]. Stephen Hawking, “ A Brief History of Relativity”, Time Magazine, Vol.154, No 27, 1999

[2]. Stephen Hawking, "A Brief History of Time”, Bantam Dell Publishing, 1988

[3]. Richard P Feynman "Surely you are joking Mr. Feynman" W.W Norton \& Company, 1985 
[4]. Brian Greene " The Elegant Universe", W.W Norton \& Company, 2003

[5]. Gordon Fraser "The New Physics for the twenty-first century" Cambridge University Press, UK, 2006

[6]. Art Hobson, "Physics: Concepts and Connections", Pearson Education Limited, 2013.

[7]. https://science.nasa.gov/astrophysics/focus-areas/what-is-dark-energy 